\title{
A New Hybrid Algorithm for Variational Inclusions, Generalized Equilibrium Problems, and a Finite Family of Quasi-Nonexpansive Mappings
}

\author{
Prasit Cholamjiak and Suthep Suantai \\ Department of Mathematics, Faculty of Science, Chiang Mai University, Chiang Mai 50200, Thailand \\ Correspondence should be addressed to Suthep Suantai, scmti005@chiangmai.ac.th
}

Received 12 June 2009; Accepted 28 September 2009

Recommended by Naseer Shahzad

We proposed in this paper a new iterative scheme for finding common elements of the set of fixed points of a finite family of quasi-nonexpansive mappings, the set of solutions of variational inclusion, and the set of solutions of generalized equilibrium problems. Some strong convergence results were derived by using the concept of $W$-mappings for a finite family of quasi-nonexpansive mappings. Strong convergence results are derived under suitable conditions in Hilbert spaces.

Copyright (c) 2009 P. Cholamjiak and S. Suantai. This is an open access article distributed under the Creative Commons Attribution License, which permits unrestricted use, distribution, and reproduction in any medium, provided the original work is properly cited.

\section{Introduction}

Let $H$ be a real Hilbert space with inner product $\langle\cdot, \cdot\rangle$ and inducted norm $\|\cdot\|$, and let $C$ be a nonempty closed and convex subset of $H$. Then, a mapping $T: C \rightarrow C$ is said to be

(1) nonexpansive if $\|T x-T y\| \leq\|x-y\|$, for all $x, y \in C$;

(2) quasi-nonexpansive if $\|T x-p\| \leq\|x-p\|$, for all $x \in C$ and $p \in F(T)$;

(3) L-Lipschitzian if there exists a constant $L>0$ such that $\|T x-T y\| \leq L\|x-y\|$, for all $x, y \in C$. We denoted by $F(T)$ the set of fixed points of $T$.

In 1953, Mann [1] introduced the following iterative procedure to approximate a fixed point of a nonexpansive mapping $T$ in a Hilbert space $H$ :

$$
x_{n+1}=\alpha_{n} x_{n}+\left(1-\alpha_{n}\right) T x_{n}, \quad \forall n \in \mathbf{N},
$$

where the initial point $x_{0}$ is taken in $C$ arbitrarily and $\left\{\alpha_{n}\right\}$ is a sequence in $[0,1]$. 
However, we note that Mann's iteration process (1.1) has only weak convergence, in general; for instance, see $[2,3]$.

Many authors attempt to modify the process (1.1) so that strong convergence is guaranteed that has recently been made. Nakajo and Takahashi [4] proposed the following modification which is the so-called CQ method and proved the following strong convergence theorem for a nonexpansive mapping $T$ in a Hilbert space $H$.

Theorem 1.1 (see [4]). Let $C$ be a nonempty closed convex subset of a Hilbert space $H$ and let $T$ be a nonexpansive mapping of $C$ into itself such that $F(T) \neq \emptyset$. Suppose that $x_{1}=x \in C$ and $\left\{x_{n}\right\}$ is given by

$$
\begin{aligned}
& y_{n}=\alpha_{n} x_{n}+\left(1-\alpha_{n}\right) T x_{n}, \\
& C_{n}=\left\{z \in C:\left\|y_{n}-z\right\| \leq\left\|x_{n}-z\right\|\right\}, \\
& Q_{n}=\left\{z \in C:\left\langle x_{n}-z, x-x_{n}\right\rangle \geq 0\right\}, \\
& x_{n+1}=P_{C_{n} \cap Q_{n}} x, \quad \forall n \in \mathbf{N},
\end{aligned}
$$

where $0 \leq \alpha_{n} \leq a<1$. Then, $\left\{x_{n}\right\}$ converges strongly to $z_{0}=P_{F(T)} x$.

Let $\varphi: H \rightarrow R \cup\{\infty\}$ be a function and let $F$ be a bifunction from $C \times C$ to $R$ such that $C \cap \operatorname{dom} \varphi \neq \emptyset$, where $R$ is the set of real numbers and $\operatorname{dom} \varphi=\{x \in H: \varphi(x)<\infty\}$. The generalized equilibrium problem is to find $\widehat{x} \in C$ such that

$$
F(\widehat{x}, y)+\varphi(y)-\varphi(\widehat{x}) \geq 0, \quad \forall y \in C
$$

The set of solutions of (1.3) is denoted by $\operatorname{GEP}(F, \varphi)$; see also [5-7].

If $\varphi: H \rightarrow R \cup\{\infty\}$ is replaced by a real-valued function $\phi: C \rightarrow R$, problem (1.3) reduces to the following mixed equilibrium problem introduced by Ceng and Yao [8]: find $\hat{x} \in C$ such that

$$
F(\widehat{x}, y)+\phi(y)-\phi(\widehat{x}) \geq 0, \quad \forall y \in C
$$

Let $\varphi(x)=\delta_{C}(x)$, for all $x \in H$. Here $\delta_{C}$ denotes the indicator function of the set $C$; that is, $\delta_{C}(x)=0$ if $x \in C$ and $\delta_{C}(x)=\infty$ otherwise. Then problem (1.3) reduces to the following equilibrium problem: find $\widehat{x} \in C$ such that

$$
F(\widehat{x}, y) \geq 0, \quad \forall y \in C
$$

The set of solutions (1.5) is denoted by $\mathrm{EP}(F)$. Problem (1.5) includes, as special cases, the optimization problem, the variational inequality problem, the fixed point problem, the nonlinear complementarity problem, the Nash equilibrium problem in noncooperative games, and the vector optimization problem; see [9-12] and the reference cited therein.

Recently, Tada and Takahashi [13] proposed a new iteration for finding a common element of the set of solutions of an equilibrium problem and the set of fixed points of a nonexpansive mapping $T$ in a Hilbert space $H$ and then obtain the following theorem. 
Theorem 1.2 (see [13]). Let $H$ be a real Hilbert space, let $C$ be a closed convex subset of $H$, let $F: C \times C \rightarrow R$ be a bifunction, and let $T: C \rightarrow C$ be a nonexpansive mapping such that $F(T) \cap$ $E P(F) \neq \emptyset$. For an initial point $x_{1}=x \in C$, let a sequence $\left\{x_{n}\right\}$ be generated by

$$
\begin{aligned}
& F\left(u_{n}, y\right)+\frac{1}{r_{n}}\left\langle y-u_{n}, u_{n}-x_{n}\right\rangle \geq 0 \quad \forall y \in C, \\
& y_{n}=\alpha_{n} x_{n}+\left(1-\alpha_{n}\right) T u_{n}, \\
& C_{n}=\left\{z \in C:\left\|y_{n}-z\right\| \leq\left\|x_{n}-z\right\|\right\}, \\
& Q_{n}=\left\{z \in C:\left\langle x_{n}-z, x_{n}-x\right\rangle \leq 0\right\}, \\
& x_{n+1}=P_{C_{n} \cap Q_{n}} x, \quad \forall n \in \mathbf{N},
\end{aligned}
$$

where $0 \leq \alpha_{n} \leq a<1$ and $\liminf _{n \rightarrow \infty} r_{n}>0$. Then, $\left\{x_{n}\right\}$ converges strongly to $P_{F(T) \cap E P(F)} x$.

Let $A: H \rightarrow H$ be a single-valued nonlinear mapping and let $M: H \rightarrow 2^{H}$ be a set-valued mapping. The variational inclusion is to find $\widehat{x} \in H$ such that

$$
\theta \in A(\widehat{x})+M(\widehat{x})
$$

where $\theta$ is the zero vector in $H$. The set of solutions of problem (1.7) is denoted by $I(A, M)$. Recall that a mapping $A: H \rightarrow H$ is called $\alpha$-inverse strongly monotone if there exists a constant $\alpha>0$ such that

$$
\langle A x-A y, x-y\rangle \geq \alpha\|A x-A y\|^{2}, \quad \forall x, y \in H .
$$

A set-valued mapping $M: H \rightarrow 2^{H}$ is called monotone if for all $x, y \in H, f \in M(x)$, and $g \in M(y)$ imply $\langle x-y, f-g\rangle \geq 0$. A monotone mapping $M$ is maximal if its graph $G(M):=\{(f, x) \in H \times H: f \in M(x)\}$ of $M$ is not properly contained in the graph of any other monotone mapping. It is known that a monotone mapping $M$ is maximal if and only if for $(x, f) \in H \times H,\langle x-y, f-g\rangle \geq 0$ for all $(y, g) \in G(M)$ imply $f \in M(x)$. We define the resolvent operator $J_{M, \lambda}$ associated with $M$ and $\lambda$ as follows:

$$
J_{M, \lambda}(x)=(I+\lambda M)^{-1}(x), \quad x \in H, \lambda>0 .
$$

It is known that the resolvent operator $J_{M, \lambda}$ is single-valued, nonexpansive, and 1inverse strongly monotone; see [14], and that a solution of problem (1.7) is a fixed point of the operator $J_{M, \lambda}(I-\lambda A)$ for all $\lambda>0$; see also [15]. If $0<\lambda<2 \alpha$, it is easy to see that $J_{M, \lambda}(I-\lambda A)$ is a nonexpansive mapping; consequently, $I(A, M)$ is closed and convex.

The equilibrium problems, generalized equilibrium problems, variational inequality problems, and variational inclusions have been intensively studied by many authors; for instance, see $[8,16-43]$.

Motivated by Tada and Takahashi [13] and Peng et al. [7], we introduce a new approximation scheme for finding a common element of the set of fixed points of a finite family of quasi-nonexpansive and Lipschitz mappings, the set of solutions of a generalized 
equilibrium problem, and the set of solutions of a variational inclusion with set-valued maximal monotone and inverse strongly monotone mappings in the framework of Hilbert spaces.

\section{Preliminaries and Lemmas}

Let $C$ be a closed convex subset of a real Hilbert space $H$ with norm $\|\cdot\|$ and inner product $\langle\cdot, \cdot\rangle$. For each $x \in H$, there exists a unique nearest point in $C$, denoted by $P_{C} x$, such that $\left\|x-P_{C} x\right\|=\min _{y \in C}\|x-y\| . P_{C}$ is called the metric projection of $H$ on to $C$. It is also known that for $x \in H$ and $z \in C, z=P_{C} x$ is equivalent to $\langle x-z, y-z\rangle \leq 0$ for all $y \in C$. Furthermore

$$
\left\|y-P_{C} x\right\|^{2}+\left\|x-P_{C} x\right\|^{2} \leq\|x-y\|^{2}
$$

for all $x \in H, y \in C$; see also $[4,44]$. In a real Hilbert space, we also know that

$$
\|\lambda x+(1-\lambda) y\|^{2}=\lambda\|x\|^{2}+(1-\lambda)\|y\|^{2}-\lambda(1-\lambda)\|x-y\|^{2}
$$

for all $x, y \in H$ and $\lambda \in[0,1]$.

Lemma 2.1 (see [45]). Let $C$ be a nonempty closed convex subset of a Hilbert space $H$. Then for points $w, x, y \in H$ and a real number $a \in R$, the set

$$
D:=\left\{z \in C:\|y-z\|^{2} \leq\|x-z\|^{2}+\langle w, z\rangle+a\right\} \text { is closed and convex. }
$$

For solving the generalized equilibrium problem, let us give the following assumptions for $F, \varphi$, and the set $C$ :

(A1) $F(x, x)=0$ for all $x \in C$;

(A2) $F$ is monotone, that is, $F(x, y)+F(y, x) \leq 0$ for all $x, y \in C$;

(A3) for each $y \in C, x \mapsto F(x, y)$ is weakly upper semicontinuous;

(A4) for each $x \in C, y \mapsto F(x, y)$ is convex;

(A5) for each $x \in C, y \mapsto F(x, y)$ is lower semicontinuous;

(B1) for each $x \in H$ and $r>0$, there exists a bounded subset $D_{x} \subseteq C$ and $y_{x} \in C \cap \operatorname{dom} \varphi$ such that for any $z \in C \backslash D_{x}$,

$$
F\left(z, y_{x}\right)+\varphi\left(y_{x}\right)+\frac{1}{r}\left\langle y_{x}-z, z-x\right\rangle<\varphi(z)
$$

(B2) $C$ is a bounded set.

Lemma 2.2 (see [7]). Let $C$ be a nonempty closed convex subset of a real Hilbert $H$. Let $F$ be a bifunction from $C \times C$ to $R$ satisfying $(A 1)-(A 5)$ and let $\varphi: H \rightarrow R \cup\{\infty\}$ be a proper lower 
semicontinuous and convex function such that $C \cap \operatorname{dom} \varphi \neq \emptyset$. For $r>0$ and $x \in H$, define a mapping $S_{r}: H \rightarrow C$ as follows:

$$
S_{r}(x)=\left\{z \in C: F(z, y)+\varphi(y)+\frac{1}{r}\langle y-z, z-x\rangle \geq \varphi(z), \forall y \in C\right\}
$$

Assume that either (B1) or (B2) holds. Then, the following conclusions hold:

(1) for each $x \in H, S_{r}(x) \neq \emptyset$;

(2) $S_{r}$ is single-valued;

(3) $S_{r}$ is firmly nonexpansive, that is, for any $x, y \in H$,

$$
\left\|S_{r}(x)-S_{r}(y)\right\|^{2} \leq\left\langle S_{r}(x)-S_{r}(y), x-y\right\rangle
$$

(4) $F\left(S_{r}\right)=\operatorname{GEP}(F, \varphi)$;

(5) $\operatorname{GEP}(F, \varphi)$ is closed and convex.

Lemma 2.3 (see [14]). Let $M: H \rightarrow 2^{H}$ be a maximal monotone mapping and let $A: H \rightarrow H$ be a Lipshitz continuous mapping. Then the mapping $S=M+A: H \rightarrow 2^{H}$ is a maximal monotone mapping.

Lemma 2.4. Let $C$ be a nonempty closed convex subset of a real Hilbert space $H$. Let $T$ be a quasinonexpansive and L-Lipschitz mapping of $C$ into itself. Then, $F(T)$ is closed and convex.

Proof. Since $T$ is $L$-Lipschitz, it is easy to show that $F(T)$ is closed.

Let $x, y \in F(T)$ and $z=t x+(1-t) y$ where $t \in(0,1)$. From (2.2), we have

$$
\begin{aligned}
\|z-T z\|^{2} & =t\|x-T z\|^{2}+(1-t)\|y-T z\|^{2}-t(1-t)\|x-y\|^{2} \\
& \leq t\|x-z\|^{2}+(1-t)\|y-z\|^{2}-t(1-t)\|x-y\|^{2} \\
& =t(1-t)^{2}\|x-y\|^{2}+(1-t) t^{2}\|x-y\|^{2}-t(1-t)\|x-y\|^{2}=0,
\end{aligned}
$$

which implies $z \in F(T)$; consequently, $F(T)$ is convex. This completes the proof.

Lemma 2.5 (see [46]). In a strictly convex Banach space X, if

$$
\|x\|=\|y\|=\|\lambda x+(1-\lambda) y\|
$$

for all $x, y \in X$ and $\lambda \in(0,1)$, then $x=y$. 
In 1999, Atsushiba and Takahashi [47] introduced the concept of the $W$-mapping as follows:

$$
\begin{aligned}
U_{1} & =\beta_{1} T_{1}+\left(1-\beta_{1}\right) I, \\
U_{2} & =\beta_{2} T_{2} U_{1}+\left(1-\beta_{2}\right) I, \\
& \vdots \\
U_{N-1} & =\beta_{N-1} T_{N-1} U_{N-2}+\left(1-\beta_{N-1}\right) I, \\
W & =U_{N}=\beta_{N} T_{N} U_{N-1}+\left(1-\beta_{N}\right) I,
\end{aligned}
$$

where $\left\{T_{i}\right\}_{i=1}^{N}$ is a finite mapping of $C$ into itself and $\beta_{i} \in[0,1]$ for all $i=1,2, \ldots, N$ with $\sum_{i=1}^{N} \beta_{i}=1$.

Such a mapping $W$ is called the $W$-mapping generated by $T_{1}, T_{2}, \ldots, T_{N}$ and $\beta_{1}, \beta_{2}, \ldots, \beta_{N}$; see also [48-50]. Throughout this paper, we denote $F:=\bigcap_{i=1}^{N} F\left(T_{i}\right)$.

Next, we prove some useful lemmas concerning the $W$-mapping.

Lemma 2.6. Let $C$ be a nonempty closed convex subset of a strictly convex Banach space X. Let $\left\{T_{i}\right\}_{i=1}^{N}$ be a finite family of quasi-nonexpansive and $L_{i}$-Lipschitz mappings of $C$ into itself such that $F:=$ $\bigcap_{i=1}^{N} F\left(T_{i}\right) \neq \emptyset$ and let $\beta_{1}, \beta_{2}, \ldots, \beta_{N}$ be real numbers such that $0<\beta_{i}<1$ for all $i=1,2, \ldots, N-1,0<$ $\beta_{N} \leq 1$, and $\sum_{i=1}^{N} \beta_{i}=1$. Let $W$ be the $W$-mapping generated by $T_{1}, T_{2}, \ldots, T_{N}$ and $\beta_{1}, \beta_{2}, \ldots, \beta_{N}$. Then, the followings hold:

(i) $W$ is quasi-nonexpansive and Lipschitz;

(ii) $F(W)=\bigcap_{i=1}^{N} F\left(T_{i}\right)$.

Proof. (i) For each $x \in C$ and $z \in F$, we observe that

$$
\left\|T_{1} x-z\right\| \leq\|x-z\|
$$

Let $k \in\{2,3, \ldots, N\}$, then

$$
\begin{aligned}
\left\|U_{k} x-z\right\| & =\left\|\beta_{k} T_{k} U_{k-1} x+\left(1-\beta_{k}\right) x-z\right\| \\
& \leq \beta_{k}\left\|U_{k-1} x-z\right\|+\left(1-\beta_{k}\right)\|x-z\| .
\end{aligned}
$$

Hence,

$$
\begin{aligned}
\|W x-z\| & =\left\|U_{N} x-z\right\| \\
& \leq \beta_{N}\left\|U_{N-1} x-z\right\|+\left(1-\beta_{N}\right)\|x-z\| \\
& \leq \beta_{N}\left(\beta_{N-1}\left\|U_{N-2} x-z\right\|+\left(1-\beta_{N-1}\right)\|x-z\|\right)+\left(1-\beta_{N}\right)\|x-z\|
\end{aligned}
$$


Fixed Point Theory and Applications

$$
\begin{aligned}
& \leq \beta_{N}\left(\beta_{N-1}\left(\beta_{N-2}\left\|U_{N-3} x-z\right\|+\left(1-\beta_{N-2}\right)\|x-z\|\right)+\left(1-\beta_{N-1}\right)\|x-z\|\right) \\
& +\left(1-\beta_{N}\right)\|x-z\| \\
& \vdots \\
& \leq \beta_{N}\left(\beta _ { N - 1 } \left(\beta_{N-2} \cdots\left(\beta_{2}\left(\beta_{1}\left\|T_{1} x-z\right\|+\left(1-\beta_{1}\right)\|x-z\|\right)+\left(1-\beta_{2}\right)\|x-z\|\right)\right.\right. \\
& \left.\left.+\cdots+\left(1-\beta_{N-2}\right)\|x-z\|\right)+\left(1-\beta_{N-1}\right)\|x-z\|\right)+\left(1-\beta_{N}\right)\|x-z\| \\
& \leq \beta_{N}\left(\beta _ { N - 1 } \left(\beta_{N-2} \cdots\left(\beta_{2}\left(\beta_{1}\|x-z\|+\left(1-\beta_{1}\right)\|x-z\|\right)+\left(1-\beta_{2}\right)\|x-z\|\right)\right.\right. \\
& \left.\left.+\cdots+\left(1-\beta_{N-2}\right)\|x-z\|\right)+\left(1-\beta_{N-1}\right)\|x-z\|\right)+\left(1-\beta_{N}\right)\|x-z\| \\
& =\beta_{N}\left(\beta _ { N - 1 } \left(\beta_{N-2} \cdots\left(\beta_{3}\left(\beta_{2}\|x-z\|+\left(1-\beta_{2}\right)\|x-z\|\right)+\left(1-\beta_{3}\right)\|x-z\|\right)\right.\right. \\
& \left.\left.+\cdots+\left(1-\beta_{N-2}\right)\|x-z\|\right)+\left(1-\beta_{N-1}\right)\|x-z\|\right)+\left(1-\beta_{N}\right)\|x-z\| \\
& =\|x-z\| .
\end{aligned}
$$

This shows that $W$ is a quasi-nonexpansive mapping.

Next, we claim that $W$ is a Lipschitz mapping. Note that $T_{i}$ is $L_{i}$-Lipschitz for all $i=$ $1,2, \ldots, N$. For each $x, y \in C$, we observe

$$
\begin{aligned}
\left\|U_{1} x-U_{1} y\right\| & =\left\|\beta_{1} T_{1} x+\left(1-\beta_{1}\right) x-\beta_{1} T_{1} y-\left(1-\beta_{1}\right) y\right\| \\
& \leq \beta_{1}\left\|T_{1} x-T_{1} y\right\|+\left(1-\beta_{1}\right)\|x-y\| \\
& \leq\left(\beta_{1} L_{1}+\left(1-\beta_{1}\right)\right)\|x-y\| .
\end{aligned}
$$

Let $k \in\{2,3, \ldots, N\}$, then

$$
\begin{aligned}
\left\|U_{k} x-U_{k} y\right\| & =\left\|\beta_{k} T_{k} U_{k-1} x+\left(1-\beta_{k}\right) x-\beta_{k} T_{k} U_{k-1} y-\left(1-\beta_{k}\right) y\right\| \\
& \leq \beta_{k} L_{k}\left\|U_{k-1} x-U_{k-1} y\right\|+\left(1-\beta_{k}\right)\|x-y\| .
\end{aligned}
$$

Hence,

$$
\begin{aligned}
\|W x-W y\| \leq & \beta_{N} L_{N}\left\|U_{N-1} x-U_{N-1} y\right\|+\left(1-\beta_{N}\right)\|x-y\| \\
\leq & \beta_{N} L_{N} \beta_{N-1} L_{N-1}\left\|U_{N-2} x-U_{N-2} y\right\| \\
& +\left(\beta_{N} L_{N}\left(1-\beta_{N-1}\right)+\left(1-\beta_{N}\right)\right)\|x-y\|
\end{aligned}
$$




$$
\begin{aligned}
\leq & \beta_{N} L_{N} \beta_{N-1} L_{N-1} \cdots \beta_{2} L_{2}\left\|U_{1} x-U_{1} y\right\| \\
+ & \left(\beta_{N} L_{N} \beta_{N-1} L_{N-1} \cdots \beta_{3} L_{3}\left(1-\beta_{2}\right)\right. \\
& +\beta_{N} L_{N} \beta_{N-1} L_{N-1} \cdots \beta_{4} L_{4}\left(1-\beta_{3}\right) \\
& \left.+\cdots+\beta_{N} L_{N}\left(1-\beta_{N-1}\right)+\left(1-\beta_{N}\right)\right)\|x-y\| \\
\leq & \beta_{N} L_{N} \beta_{N-1} L_{N-1} \cdots \beta_{2} L_{2}\left(\beta_{1} L_{1}+\left(1-\beta_{1}\right)\|x-y\|\right) \\
+ & \left(\beta_{N} L_{N} \beta_{N-1} L_{N-1} \cdots \beta_{3} L_{3}\left(1-\beta_{2}\right)\right. \\
& +\beta_{N} L_{N} \beta_{N-1} L_{N-1} \cdots \beta_{4} L_{4}\left(1-\beta_{3}\right) \\
& \left.+\cdots+\beta_{N} L_{N}\left(1-\beta_{N-1}\right)+\left(1-\beta_{N}\right)\right)\|x-y\| \\
=( & \beta_{N} L_{N} \beta_{N-1} L_{N-1} \cdots \beta_{1} L_{1} \\
& +\beta_{N} L_{N} \beta_{N-1} L_{N-1} \cdots \beta_{2} L_{2}\left(1-\beta_{1}\right) \\
& +\beta_{N} L_{N} \beta_{N-1} L_{N-1} \cdots \beta_{3} L_{3}\left(1-\beta_{2}\right) \\
& +\beta_{N} L_{N} \beta_{N-1} L_{N-1} \cdots \beta_{4} L_{4}\left(1-\beta_{3}\right) \\
& \left.+\cdots+\beta_{N} L_{N}\left(1-\beta_{N-1}\right)+\left(1-\beta_{N}\right)\right)\|x-y\| . \\
\leq & \left(L_{N} L_{N-1} \cdots L_{1}+L_{N} L_{N-1} \cdots L_{2}+L_{N} L_{N-1} \cdots L_{3}\right. \\
& \left.+L_{N} L_{N-1} \cdots L_{4}+\cdots+L_{N} L_{N-1}+L_{N}+1\right)\|x-y\| .
\end{aligned}
$$

Since $L_{i}>0$ for all $i=1,2, \ldots, N$, we get that $W$ is a Lipschitz mapping.

(ii) Since $F \subset F(W)$ is trivial, it suffices to show that $F(W) \subset F$. To end this, let $p \in$ $F(W)$ and $x^{*} \in F$. Then, we have

$$
\begin{aligned}
\left\|p-x^{*}\right\| & =\left\|W p-x^{*}\right\|=\left\|\beta_{N}\left(T_{N} U_{N-1} p-x^{*}\right)+\left(1-\beta_{N}\right)\left(p-x^{*}\right)\right\| \\
& \leq \beta_{N}\left\|U_{N-1} p-x^{*}\right\|+\left(1-\beta_{N}\right)\left\|p-x^{*}\right\| \\
& =\beta_{N}\left\|\beta_{N-1}\left(T_{N-1} U_{N-2} p-x^{*}\right)+\left(1-\beta_{N-1}\right)\left(p-x^{*}\right)\right\|+\left(1-\beta_{N}\right)\left\|p-x^{*}\right\| \\
& \leq \beta_{N} \beta_{N-1}\left\|U_{N-2} p-x^{*}\right\|+\left(1-\beta_{N} \beta_{N-1}\right)\left\|p-x^{*}\right\| \\
& =\beta_{N} \beta_{N-1}\left\|\beta_{N-2}\left(T_{N-2} U_{N-3} p-x^{*}\right)+\left(1-\beta_{N-2}\right)\left(p-x^{*}\right)\right\|+\left(1-\beta_{N} \beta_{N-1}\right)\left\|p-x^{*}\right\| \\
& \leq \beta_{N} \beta_{N-1} \beta_{N-2}\left\|U_{N-3} p-x^{*}\right\|+\left(1-\beta_{N} \beta_{N-1} \beta_{N-2}\right)\left\|p-x^{*}\right\| \\
& \vdots \\
& =\beta_{N} \beta_{N-1} \cdots \beta_{3}\left\|\beta_{2}\left(T_{2} U_{1} p-x^{*}\right)+\left(1-\beta_{2}\right)\left(p-x^{*}\right)\right\|+\left(1-\beta_{N} \beta_{N-1} \cdots \beta_{3}\right)\left\|p-x^{*}\right\|
\end{aligned}
$$


Fixed Point Theory and Applications

$$
\begin{aligned}
& \leq \beta_{N} \beta_{N-1} \cdots \beta_{2}\left\|T_{2} U_{1} p-x^{*}\right\|+\left(1-\beta_{N} \beta_{N-1} \cdots \beta_{2}\right)\left\|p-x^{*}\right\| \\
& \leq \beta_{N} \beta_{N-1} \cdots \beta_{2}\left\|U_{1} p-x^{*}\right\|+\left(1-\beta_{N} \beta_{N-1} \cdots \beta_{2}\right)\left\|p-x^{*}\right\| \\
& =\beta_{N} \beta_{N-1} \cdots \beta_{2}\left\|\beta_{1}\left(T_{1} p-x^{*}\right)+\left(1-\beta_{1}\right)\left(p-x^{*}\right)\right\|+\left(1-\beta_{N} \beta_{N-1} \cdots \beta_{2}\right)\left\|p-x^{*}\right\| \\
& \leq \beta_{N} \beta_{N-1} \cdots \beta_{2} \beta_{1}\left\|T_{1} p-x^{*}\right\|+\left(1-\beta_{N} \beta_{N-1} \cdots \beta_{2} \beta_{1}\right)\left\|p-x^{*}\right\| \\
& \leq \beta_{N} \beta_{N-1} \cdots \beta_{2} \beta_{1}\left\|p-x^{*}\right\|+\left(1-\beta_{N} \beta_{N-1} \cdots \beta_{2} \beta_{1}\right)\left\|p-x^{*}\right\|=\left\|p-x^{*}\right\| .
\end{aligned}
$$

This shows that

$$
\left\|p-x^{*}\right\|=\beta_{N} \beta_{N-1} \cdots \beta_{2}\left\|\beta_{1}\left(T_{1} p-x^{*}\right)+\left(1-\beta_{1}\right)\left(p-x^{*}\right)\right\|+\left(1-\beta_{N} \beta_{N-1} \cdots \beta_{2}\right)\left\|p-x^{*}\right\|,
$$

and hence

$$
\left\|p-x^{*}\right\|=\left\|\beta_{1}\left(T_{1} p-x^{*}\right)+\left(1-\beta_{1}\right)\left(p-x^{*}\right)\right\| .
$$

Again by (2.16), we see that $\left\|p-x^{*}\right\|=\left\|T_{1} p-x^{*}\right\|$. Hence

$$
\left\|p-x^{*}\right\|=\left\|T_{1} p-x^{*}\right\|=\left\|\beta_{1}\left(T_{1} p-x^{*}\right)+\left(1-\beta_{1}\right)\left(p-x^{*}\right)\right\| .
$$

Applying Lemma 2.5 to (2.19), we get that $T_{1} p=p$ and hence $U_{1} p=p$.

Again by (2.16), we have

$$
\left\|p-x^{*}\right\|=\beta_{N} \beta_{N-1} \cdots \beta_{3}\left\|\beta_{2}\left(T_{2} U_{1} p-x^{*}\right)+\left(1-\beta_{2}\right)\left(p-x^{*}\right)\right\|+\left(1-\beta_{N} \beta_{N-1} \cdots \beta_{3}\right)\left\|p-x^{*}\right\|,
$$

and hence

$$
\left\|p-x^{*}\right\|=\left\|\beta_{2}\left(T_{2} U_{1} p-x^{*}\right)+\left(1-\beta_{2}\right)\left(p-x^{*}\right)\right\| .
$$

From (2.16), we know that $\left\|U_{1} p-x^{*}\right\|=\left\|T_{2} U_{1} p-x^{*}\right\|$. Since $U_{1} p=p$, we have

$$
\left\|p-x^{*}\right\|=\left\|T_{2} p-x^{*}\right\|=\left\|\beta_{2}\left(T_{2} p-x^{*}\right)+\left(1-\beta_{2}\right)\left(p-x^{*}\right)\right\| .
$$

Applying Lemma 2.5 to (2.22), we get that $T_{2} p=p$ and hence $U_{2} p=p$.

By proving in the same manner, we can conclude that $T_{i} p=p$ and $U_{i} p=p$ for all $i=1,2, \ldots, N-1$. Finally, we also have

$$
\left\|p-T_{N} p\right\| \leq\|p-W p\|+\left\|W p-T_{N} p\right\|=\|p-W p\|+\left(1-\beta_{N}\right)\left\|p-T_{N} p\right\|
$$

which yields that $p=T_{N} p$ since $p \in F(W)$. Hence $p \in F:=\bigcap_{i=1}^{N} F\left(T_{i}\right)$. 
Lemma 2.7. Let $C$ be a nonempty closed convex subset of a Banach space $X$. Let $\left\{T_{i}\right\}_{i=1}^{N}$ be a finite family of quasi-nonexpansive and $L_{i}$-Lipschitz mappings of $C$ into itself and $\left\{\beta_{n, i}\right\}_{i=1}^{N}$ sequences in $[0,1]$ such that $\beta_{n, i} \rightarrow \beta_{i}$ as $n \rightarrow \infty$. Moreover, for every $n \in \mathbf{N}$, let $W$ and $W_{n}$ be the $W$-mappings generated by $T_{1}, T_{2}, \ldots, T_{N}$ and $\beta_{1}, \beta_{2}, \ldots, \beta_{N}$ and $T_{1}, T_{2}, \ldots, T_{N}$ and $\beta_{n, 1}, \beta_{n, 2}, \ldots, \beta_{n, N}$, respectively. Then

$$
\lim _{n \rightarrow \infty}\left\|W_{n} x-W x\right\|=0, \quad \forall x \in C
$$

Proof. Let $x \in C$ and $U_{k}$ and $U_{n, k}$ be generated by $T_{1}, T_{2}, \ldots, T_{k}$ and $\beta_{1}, \beta_{2}, \ldots, \beta_{k}$ and $T_{1}, T_{2}, \ldots, T_{k}$ and $\beta_{n, 1}, \beta_{n, 2}, \ldots, \beta_{n, k}$, respectively. Then

$$
\left\|U_{n, 1} x-U_{1} x\right\|=\left\|\left(\beta_{n, 1}-\beta_{1}\right)\left(T_{1} x-x\right)\right\| \leq\left|\beta_{n, 1}-\beta_{1}\right|\left\|T_{1} x-x\right\| .
$$

Let $k \in\{2,3, \ldots, N\}$ and $M=\max \left\{\left\|T_{k} U_{k-1} x\right\|+\|x\|: k=2,3, \ldots, N\right\}$. Then

$$
\begin{aligned}
\left\|U_{n, k} x-U_{k} x\right\| & =\left\|\beta_{n, k} T_{k} U_{n, k-1} x+\left(1-\beta_{n, k}\right) x-\beta_{k} T_{k} U_{k-1}-\left(1-\beta_{k}\right) x\right\| \\
& =\left\|\beta_{n, k} T_{k} U_{n, k-1} x-\beta_{n, k} x-\beta_{k} T_{k} U_{k-1}+\beta_{k} x\right\| \\
& \leq \beta_{n, k}\left\|T_{k} U_{n, k-1} x-T_{k} U_{k-1} x\right\|+\left|\beta_{n, k}-\beta_{k}\right|\left\|T_{k} U_{k-1} x\right\|+\left|\beta_{n, k}-\beta_{k}\right|\|x\| \\
& \leq L_{k}\left\|U_{n, k-1} x-U_{k-1} x\right\|+\left|\beta_{n, k}-\beta_{k}\right| M .
\end{aligned}
$$

It follows that

$$
\begin{aligned}
\left\|W_{n} x-W x\right\|= & \left\|U_{n, N} x-U_{N} x\right\| \\
\leq & L_{N}\left\|U_{n, N-1} x-U_{N-1} x\right\|+\left|\beta_{n, N}-\beta_{N}\right| M \\
\leq & L_{N}\left(L_{N-1}\left\|U_{n, N-2} x-U_{N-2} x\right\|+\left|\beta_{n, N-1}-\beta_{N-1}\right| M\right)+\left|\beta_{n, N}-\beta_{N}\right| M \\
= & L_{N} L_{N-1}\left\|U_{n, N-2} x-U_{N-2} x\right\|+L_{N}\left|\beta_{n, N-1}-\beta_{N-1}\right| M+\left|\beta_{n, N}-\beta_{N}\right| M \\
& \vdots \\
\leq & L_{N} L_{N-1} \cdots L_{3}\left(L_{2}\left\|U_{n, 1} x-U_{1} x\right\|+\left|\beta_{n, 2}-\beta_{2}\right| M\right) \\
& +L_{N} L_{N-1} \cdots L_{4}\left|\beta_{n, 3}-\beta_{3}\right| M+\cdots+L_{N}\left|\beta_{n, N-1}-\beta_{N-1}\right| M+\left|\beta_{n, N}-\beta_{N}\right| M \\
\leq & L_{N} L_{N-1} \cdots L_{2}\left|\beta_{n, 1}-\beta_{1}\right|\left|T_{1} x-x \|+L_{N} L_{N-1} \cdots L_{3}\right| \beta_{n, 2}-\beta_{2} \mid M \\
& +L_{N} L_{N-1} \cdots L_{4}\left|\beta_{n, 3}-\beta_{3}\right| M+\cdots+L_{N}\left|\beta_{n, N-1}-\beta_{N-1}\right| M+\left|\beta_{n, N}-\beta_{N}\right| M .
\end{aligned}
$$

Since $\beta_{n, i} \rightarrow \beta_{i}$ as $n \rightarrow \infty(i=1,2, \ldots, N)$, we obtain the result. 


\section{Strong Convergence Theorems}

In this section, we prove a strong convergence theorem which solves the problem of finding a common element of the set of solutions of a generalized equilibrium problem and the set of solutions of a variational inclusion and the set of common fixed points of a finite family of quasi-nonexpansive and Lipschitz mappings.

Theorem 3.1. Let $C$ be a nonempty closed convex subset of a real Hilbert space $H$, let $F: C \times C \rightarrow R$ be a bifunction satisfying (A1)-(A5), let $\varphi: C \rightarrow R \cup\{\infty\}$ be a proper lower semicontinuous and convex function, let $A: H \rightarrow H$ be an $\alpha$-inverse strongly monotone mapping, let $M: H \rightarrow 2^{H}$ be a maximal monotone mapping, and let $\left\{T_{i}\right\}_{i=1}^{N}$ be a finite family of quasi-nonexpansive and $L_{i}$-Lipschitz mappings of $C$ into itself. Assume that $\Omega:=\bigcap_{i=1}^{N} F\left(T_{i}\right) \cap G E P(F, \varphi) \cap I(A, M) \neq \emptyset$ and either (B1) or (B2) holds. Let $W_{n}$ be the $W$-mapping generated by $T_{1}, T_{2}, \ldots, T_{N}$ and $\beta_{n, 1}, \beta_{n, 2}, \ldots, \beta_{n, N}$. For an initial point $x_{0} \in H$ with $C_{1}=C$ and $x_{1}=P_{C_{1}} x_{0}$, let $\left\{x_{n}\right\},\left\{y_{n}\right\},\left\{z_{n}\right\}$, and $\left\{u_{n}\right\}$ be sequences generated by

$$
\begin{aligned}
& F\left(u_{n}, y\right)+\varphi(y)-\varphi\left(u_{n}\right)+\frac{1}{r_{n}}\left\langle y-u_{n}, u_{n}-x_{n}\right\rangle \geq 0, \quad \forall y \in C, \\
& y_{n}=\alpha_{n} x_{n}+\left(1-\alpha_{n}\right) W_{n} u_{n}, \\
& z_{n}=J_{M, \lambda_{n}}\left(y_{n}-\lambda_{n} A y_{n}\right), \\
& C_{n+1}=\left\{z \in C_{n}:\left\|z_{n}-z\right\| \leq\left\|y_{n}-z\right\| \leq\left\|x_{n}-z\right\|\right\}, \\
& x_{n+1}=P_{C_{n+1}} x_{0}, \quad \forall n \in \mathbf{N},
\end{aligned}
$$

where $\left\{\alpha_{n}\right\} \subset[0, a]$ for some $a \in[0,1),\left\{r_{n}\right\} \subset[b, \infty)$ for some $b \in(0, \infty)$ and $\left\{\lambda_{n}\right\} \subset[c, d]$ for some $c, d \in(0,2 \alpha)$.

Then, $\left\{x_{n}\right\},\left\{y_{n}\right\},\left\{z_{n}\right\}$, and $\left\{u_{n}\right\}$ converge strongly to $z_{0}=P_{\Omega} x_{0}$.

Proof. Since $0<c \leq \lambda_{n} \leq d<2 \alpha$ for all $n \in \mathbf{N}$, we get that $J_{M, \lambda_{n}}\left(I-\lambda_{n} A\right)$ is nonexpansive for all $n \in \mathbf{N}$. Hence, $\bigcap_{n=1}^{\infty} F\left(J_{M, \lambda_{n}}\left(I-\lambda_{n} A\right)\right)=I(A, M)$ is closed and convex. By Lemma 2.2(5), we know that $\operatorname{GEP}(F, \varphi)$ is closed and convex. By Lemma 2.4, we also know that $F:=\bigcap_{i=1}^{N} F\left(T_{i}\right)$ is closed and convex. Hence, $\Omega:=\bigcap_{i=1}^{N} F\left(T_{i}\right) \cap \operatorname{GEP}(F, \varphi) \cap I(A, M)$ is a nonempty closed convex set; consequently, $P_{\Omega} x_{0}$ is well defined for every $x_{0} \in H$.

Next, we divide the proof into seven steps.

Step 1. Show that $\Omega \subset C_{n}$ for all $n \in \mathbf{N}$.

By Lemma 2.1, we see that $C_{n}$ is closed and convex for all $n \in \mathbf{N}$. Hence $P_{C_{n+1}} x_{0}$ is well defined for every $x_{0} \in H, n \in \mathbf{N}$. Let $p \in \Omega$. From $u_{n}=S_{r_{n}} x_{n}$ and $p=J_{M, \lambda_{n}}\left(p-\lambda_{n} A p\right)$ for all $n \in \mathbf{N}$, we have

$$
\begin{aligned}
\left\|z_{n}-p\right\| & =\left\|J_{M, \lambda_{n}}\left(y_{n}-\lambda_{n} A y_{n}\right)-J_{M, \lambda_{n}}\left(p-\lambda_{n} A p\right)\right\| \\
& \leq\left\|y_{n}-p\right\| \\
& \leq \alpha_{n}\left\|x_{n}-p\right\|+\left(1-\alpha_{n}\right)\left\|W_{n} u_{n}-p\right\| \\
& \leq \alpha_{n}\left\|x_{n}-p\right\|+\left(1-\alpha_{n}\right)\left\|u_{n}-p\right\| \\
& =\alpha_{n}\left\|x_{n}-p\right\|+\left(1-\alpha_{n}\right)\left\|S_{r_{n}} x_{n}-S_{r_{n}} p\right\| \\
& \leq\left\|x_{n}-p\right\| .
\end{aligned}
$$

It follows that $p \in C_{n+1}$, and hence $\Omega \subset C_{n}$ for all $n \in \mathbf{N}$. 
Step 2. Show that $\lim _{n \rightarrow \infty}\left\|x_{n}-x_{0}\right\|$ exists.

Since $\Omega$ is a nonempty closed convex subset of $C$, there exists a unique element $z_{0}=$ $P_{\Omega} x_{0} \in \Omega \subset C_{n}$. From $x_{n}=P_{C_{n}} x_{0}$, we obtain

$$
\left\|x_{n}-x_{0}\right\| \leq\left\|z_{0}-x_{0}\right\|
$$

Hence $\left\{\left\|x_{n}-x_{0}\right\|\right\}$ is bounded; so are $\left\{y_{n}\right\},\left\{z_{n}\right\}$, and $\left\{u_{n}\right\}$.

Since $x_{n+1}=P_{C_{n+1}} x_{0} \in C_{n+1} \subset C_{n}$, we also have

$$
\left\|x_{n}-x_{0}\right\| \leq\left\|x_{n+1}-x_{0}\right\| .
$$

From (3.3) and (3.4), we get that $\lim _{n \rightarrow \infty}\left\|x_{n}-x_{0}\right\|$ exists.

Step 3. Show that $\left\{x_{n}\right\}$ is a Cauchy sequence.

By the construction of the set $C_{n}$, we know that $x_{m}=P_{C_{m}} x_{0} \in C_{m} \subset C_{n}$ for $m>n$. From (2.1), it follows that

$$
\left\|x_{m}-x_{n}\right\|^{2} \leq\left\|x_{m}-x_{0}\right\|^{2}-\left\|x_{n}-x_{0}\right\|^{2} \longrightarrow 0,
$$

as $m, n \rightarrow \infty$. Hence $\left\{x_{n}\right\}$ is a Cauchy sequence. By the completeness of $H$ and the closeness of $C$, we can assume that $x_{n} \rightarrow q \in C$.

Step 4. Show that $q \in F$.

From (3.5), we get

$$
\left\|x_{n+1}-x_{n}\right\| \longrightarrow 0
$$

as $n \rightarrow \infty$. Since $x_{n+1} \in C_{n+1} \subset C_{n}$, we have

$$
\left\|z_{n}-x_{n}\right\| \leq\left\|z_{n}-x_{n+1}\right\|+\left\|x_{n+1}-x_{n}\right\| \leq 2\left\|x_{n+1}-x_{n}\right\| \longrightarrow 0,
$$

as $n \rightarrow \infty$. Hence, $z_{n} \rightarrow q$ as $n \rightarrow \infty$. By the nonexpansiveness of $J_{M, \lambda_{n}}$ and the inverse strongly monotonicity of $A$, we obtain that

$$
\begin{aligned}
\left\|z_{n}-p\right\|^{2} & \leq\left\|y_{n}-\lambda_{n} A y_{n}-\left(p-\lambda_{n} A p\right)\right\|^{2} \\
& \leq\left\|y_{n}-p\right\|^{2}+\lambda_{n}\left(\lambda_{n}-2 \alpha\right)\left\|A y_{n}-A p\right\|^{2} \\
& \leq\left\|x_{n}-p\right\|^{2}+c(d-2 \alpha)\left\|A y_{n}-A p\right\|^{2} .
\end{aligned}
$$


This implies that

$$
\begin{aligned}
c(2 \alpha-d)\left\|A y_{n}-A p\right\|^{2} & \leq\left\|x_{n}-p\right\|^{2}-\left\|z_{n}-p\right\|^{2} \\
& \leq\left\|x_{n}-z_{n}\right\|\left(\left\|x_{n}-p\right\|+\left\|z_{n}-p\right\|\right) .
\end{aligned}
$$

It follows from (3.7) that

$$
\lim _{n \rightarrow \infty}\left\|A y_{n}-A p\right\|=0
$$

Since $J_{M, \lambda_{n}}$ is 1-inverse strongly monotone, we have

$$
\begin{aligned}
\left\|z_{n}-p\right\|^{2}= & \left\|J_{M, \lambda_{n}}\left(y_{n}-\lambda_{n} A y_{n}\right)-J_{M, \lambda_{n}}\left(p-\lambda_{n} A p\right)\right\|^{2} \\
\leq & \left\langle\left(y_{n}-\lambda_{n} A y_{n}\right)-\left(p-\lambda_{n} A p\right), z_{n}-p\right\rangle \\
= & \frac{1}{2}\left(\left\|\left(y_{n}-\lambda_{n} A y_{n}\right)-\left(p-\lambda_{n} A p\right)\right\|^{2}+\left\|z_{n}-p\right\|^{2}\right. \\
& \left.\quad-\left\|\left(y_{n}-\lambda_{n} A y_{n}\right)-\left(p-\lambda_{n} A p\right)-\left(z_{n}-p\right)\right\|^{2}\right) \\
\leq & \frac{1}{2}\left(\left\|y_{n}-p\right\|^{2}+\left\|z_{n}-p\right\|^{2}-\left\|\left(y_{n}-z_{n}\right)-\lambda_{n}\left(A y_{n}-A p\right)\right\|^{2}\right) \\
\leq & \frac{1}{2}\left(\left\|x_{n}-p\right\|^{2}+\left\|z_{n}-p\right\|^{2}-\left\|y_{n}-z_{n}\right\|^{2}+2 \lambda_{n}\left\langle y_{n}-z_{n}, A y_{n}-A p\right\rangle\right) \\
\leq & \frac{1}{2}\left(\left\|x_{n}-p\right\|^{2}+\left\|z_{n}-p\right\|^{2}-\left\|y_{n}-z_{n}\right\|^{2}+2 \lambda_{n}\left\|y_{n}-z_{n}\right\|\left\|A y_{n}-A p\right\|\right) .
\end{aligned}
$$

This implies that

$$
\left\|z_{n}-p\right\|^{2} \leq\left\|x_{n}-p\right\|^{2}-\left\|y_{n}-z_{n}\right\|^{2}+2 \lambda_{n}\left\|y_{n}-z_{n}\right\|\left\|A y_{n}-A p\right\| .
$$

It follows that

$$
\begin{aligned}
\left\|y_{n}-z_{n}\right\|^{2} \leq & \left\|x_{n}-z_{n}\right\|\left(\left\|x_{n}-p\right\|+\left\|z_{n}-p\right\|\right) \\
& +2 d\left\|y_{n}-z_{n}\right\|\left\|A y_{n}-A p\right\| .
\end{aligned}
$$

From (3.7) and (3.10) we get

$$
\lim _{n \rightarrow \infty}\left\|y_{n}-z_{n}\right\|=0
$$

It follows from (3.7) and (3.14) that

$$
\left\|W_{n} u_{n}-x_{n}\right\|=\frac{1}{1-\alpha_{n}}\left\|y_{n}-x_{n}\right\| \longrightarrow 0,
$$


as $n \rightarrow \infty$. Since $S_{r_{n}}$ is firmly nonexpansive and $u_{n}=S_{r_{n}} x_{n}$, we have

$$
\begin{aligned}
\left\|u_{n}-p\right\|^{2} & =\left\|S_{r_{n}} x_{n}-S_{r_{n}} p\right\|^{2} \\
& \leq\left\langle S_{r_{n}} x_{n}-S_{r_{n}} p, x_{n}-p\right\rangle \\
& =\left\langle u_{n}-p, x_{n}-p\right\rangle \\
& =\frac{1}{2}\left(\left\|u_{n}-p\right\|^{2}+\left\|x_{n}-p\right\|^{2}-\left\|x_{n}-u_{n}\right\|^{2}\right),
\end{aligned}
$$

which implies that

$$
\left\|u_{n}-p\right\|^{2} \leq\left\|x_{n}-p\right\|^{2}-\left\|x_{n}-u_{n}\right\|^{2} .
$$

It follows from (3.17) that

$$
\begin{aligned}
\left\|y_{n}-p\right\|^{2} & \leq \alpha_{n}\left\|x_{n}-p\right\|^{2}+\left(1-\alpha_{n}\right)\left\|W_{n} u_{n}-p\right\|^{2} \\
& \leq \alpha_{n}\left\|x_{n}-p\right\|^{2}+\left(1-\alpha_{n}\right)\left\|u_{n}-p\right\|^{2} \\
& \leq \alpha_{n}\left\|x_{n}-p\right\|^{2}+\left(1-\alpha_{n}\right)\left(\left\|x_{n}-p\right\|^{2}-\left\|x_{n}-u_{n}\right\|^{2}\right) \\
& =\left\|x_{n}-p\right\|^{2}-\left(1-\alpha_{n}\right)\left\|x_{n}-u_{n}\right\|^{2},
\end{aligned}
$$

which yields that

$$
(1-a)\left\|x_{n}-u_{n}\right\|^{2} \leq\left\|x_{n}-p\right\|^{2}-\left\|y_{n}-p\right\|^{2}
$$

Hence, from (3.7) and (3.14), we also have

$$
\lim _{n \rightarrow \infty}\left\|x_{n}-u_{n}\right\|=0
$$

It follows from (3.15) and (3.20) that

$$
\lim _{n \rightarrow \infty}\left\|u_{n}-W_{n} u_{n}\right\|=0
$$

By Lemma 2.7, we also get that $\lim _{n \rightarrow \infty}\left\|u_{n}-W u_{n}\right\|=0$. From Lemma 2.6(i), we know that $W$ is Lipschitz. Since $u_{n} \rightarrow q$ as $n \rightarrow \infty$, it is easy to verify that $q \in F(W)$. Moreover, by Lemma 2.6(ii), we can conclude that $q \in F:=\bigcap_{i=1}^{N} F\left(T_{i}\right)$. 
Step 5. Show that $q \in \operatorname{GEP}(F, \varphi)$.

Since $u_{n}=S_{r_{n}} x_{n}$, we have

$$
F\left(u_{n}, y\right)+\varphi(y)+\frac{1}{r_{n}}\left\langle y-u_{n}, u_{n}-x_{n}\right\rangle \geq \varphi\left(u_{n}\right), \quad \forall y \in C
$$

From (A2), we have

$$
\varphi(y)+\frac{1}{r_{n}}\left\langle y-u_{n}, u_{n}-x_{n}\right\rangle \geq F\left(y, u_{n}\right)+\varphi\left(u_{n}\right), \quad \forall y \in C
$$

It follows from (A5) and the weakly lower semicontinuity of $\varphi,\left\|x_{n}-u_{n}\right\| / r_{n} \rightarrow 0$, and $u_{n} \rightarrow q$ that

$$
F(y, q)+\varphi(q) \leq \varphi(y), \quad \forall y \in C
$$

Put $y_{t}=t y+(1-t) q$ for all $t \in(0,1]$ and $y \in C \cap \operatorname{dom} \varphi$. Since $y \in C \cap \operatorname{dom} \varphi$ and $q \in C \cap \operatorname{dom} \varphi$, we obtain $y_{t} \in C \cap \operatorname{dom} \varphi$, and hence $F\left(y_{t}, q\right)+\varphi(q) \leq \varphi\left(y_{t}\right)$. So by (A1), (A4), and the convexity of $\varphi$, we have

$$
\begin{aligned}
0 & =F\left(y_{t}, y_{t}\right)+\varphi\left(y_{t}\right)-\varphi\left(y_{t}\right) \\
& \leq t F\left(y_{t}, y\right)+(1-t) F\left(y_{t}, q\right)+t \varphi(y)+(1-t) \varphi(q)-\varphi\left(y_{t}\right) \\
& \leq t\left[F\left(y_{t}, y\right)+\varphi(y)-\varphi\left(y_{t}\right)\right]
\end{aligned}
$$

Hence,

$$
F\left(y_{t}, y\right)+\varphi(y)-\varphi\left(y_{t}\right) \geq 0
$$

Letting $t \rightarrow 0$, it follows from (A3) and the weakly semicontinuity of $\varphi$ that

$$
F(q, y)+\varphi(y) \geq \varphi(q)
$$

for all $y \in C \cap \operatorname{dom} \varphi$. Observe that if $y \in C \backslash \operatorname{dom} \varphi$, then $F(q, y)+\varphi(y) \geq \varphi(q)$ holds. Hence $q \in \operatorname{GEP}(F, \varphi)$.

Step 6. Show that $q \in I(A, M)$.

First observe that $A$ is an $(1 / \alpha)$-Lipschitz monotone mapping and $D(A)=H$. From Lemma 2.3, we know that $M+A$ is maximal monotone. Let $(v, g) \in G(M+A)$, that is, $g-A v \in M(v)$. Since $z_{n}=J_{M, \lambda_{n}}\left(y_{n}-\lambda_{n} A y_{n}\right)$, we get $y_{n}-\lambda_{n} A y_{n} \in\left(I+\lambda_{n} M\right)\left(z_{n}\right)$, that is,

$$
\frac{1}{\lambda_{n}}\left(y_{n}-z_{n}-\lambda_{n} A y_{n}\right) \in M\left(z_{n}\right)
$$


By the maximal monotonicity of $M+A$, we have

$$
\left\langle v-z_{n}, g-A v-\frac{1}{\lambda_{n}}\left(y_{n}-z_{n}-\lambda_{n} A y_{n}\right)\right\rangle \geq 0
$$

and so

$$
\begin{aligned}
\left\langle v-z_{n}, g\right\rangle & \geq\left\langle v-z_{n}, A v+\frac{1}{\lambda_{n}}\left(y_{n}-z_{n}-\lambda_{n} A y_{n}\right)\right\rangle \\
& =\left\langle v-z_{n}, A v-A z_{n}+A z_{n}-A y_{n}+\frac{1}{\lambda_{n}}\left(y_{n}-z_{n}\right)\right\rangle \\
& \geq 0+\left\langle v-z_{n}, A z_{n}-A y_{n}\right\rangle+\left\langle v-z_{n}, \frac{1}{\lambda_{n}}\left(y_{n}-z_{n}\right)\right\rangle
\end{aligned}
$$

It follows from $\left\|y_{n}-z_{n}\right\| \rightarrow 0,\left\|A y_{n}-A z_{n}\right\| \rightarrow 0$ and $z_{n} \rightarrow q$ that

$$
\lim _{n \rightarrow \infty}\left\langle v-z_{n}, g\right\rangle=\langle v-q, g\rangle \geq 0
$$

By the maximal monotonicity of $M+A$, we have $\theta \in(M+A)(q)$; consequently, $q \in I(A, M)$.

Step 7. Show that $q=z_{0}=P_{\Omega} x_{0}$.

Since $x_{n}=P_{C_{n}} x_{0}$ and $\Omega \subset C_{n}$, we obtain

$$
\left\langle x_{0}-x_{n}, x_{n}-p\right\rangle \geq 0 \quad \forall p \in \Omega
$$

By taking the limit in (3.32), we obtain

$$
\left\langle x_{0}-q, q-p\right\rangle \geq 0 \quad \forall p \in \Omega
$$

This shows that $q=P_{\Omega} x_{0}=z_{0}$.

From Steps 1-7, we can conclude that $\left\{x_{n}\right\},\left\{y_{n}\right\},\left\{z_{n}\right\}$, and $\left\{u_{n}\right\}$ converge strongly to $z_{0}=P_{\Omega} x_{0}$. This completes the proof.

\section{Applications}

As a direct consequence of Theorem 3.1, we obtain some new and interesting results in a Hilbert space as the following theorems. Recall that $\operatorname{VI}(A, C)$ is the solution set of the classical variational inequality

$$
\langle A \widehat{x}, y-\widehat{x}\rangle \geq 0, \quad \forall y \in C
$$


Theorem 4.1. Let $C$ be a nonempty closed convex subset of a real Hilbert space $H$, let $F: C \times C \rightarrow R$ be a bifunction satisfying (A1)-(A5), let $\varphi: C \rightarrow R \cup\{\infty\}$ be a proper lower semicontinuous and convex function, let $A: C \rightarrow H$ be an $\alpha$-inverse strongly monotone mapping, and let $\left\{T_{i}\right\}_{i=1}^{N}$ be a finite family of quasi-nonexpansive and $L_{i}$-Lipschitz mappings of $C$ into itself. Assume that $\Omega:=$ $\bigcap_{i=1}^{N} F\left(T_{i}\right) \cap \operatorname{GEP}(F, \varphi) \cap \operatorname{VI}(A, C) \neq \emptyset$ and either (B1) or (B2) holds. Let $W_{n}$ be the $W$-mapping generated by $T_{1}, T_{2}, \ldots, T_{N}$ and $\beta_{n, 1}, \beta_{n, 2}, \ldots, \beta_{n, N}$. For an initial point $x_{0} \in H$ with $C_{1}=C$ and $x_{1}=P_{C_{1}} x_{0}$, let $\left\{x_{n}\right\},\left\{y_{n}\right\},\left\{z_{n}\right\}$, and $\left\{u_{n}\right\}$ be sequences generated by

$$
\begin{aligned}
& F\left(u_{n}, y\right)+\varphi(y)-\varphi\left(u_{n}\right)+\frac{1}{r_{n}}\left\langle y-u_{n}, u_{n}-x_{n}\right\rangle \geq 0, \quad \forall y \in C, \\
& y_{n}=\alpha_{n} x_{n}+\left(1-\alpha_{n}\right) W_{n} u_{n}, \\
& z_{n}=P_{C}\left(y_{n}-\lambda_{n} A y_{n}\right), \\
& C_{n+1}=\left\{z \in C_{n}:\left\|z_{n}-z\right\| \leq\left\|y_{n}-z\right\| \leq\left\|x_{n}-z\right\|\right\}, \\
& x_{n+1}=P_{C_{n+1}} x_{0}, \quad \forall n \in \mathbf{N},
\end{aligned}
$$

where $\left\{\alpha_{n}\right\} \subset[0, a]$ for some $a \in[0,1),\left\{r_{n}\right\} \subset[b, \infty)$ for some $b \in(0, \infty)$, and $\left\{\lambda_{n}\right\} \subset[c, d]$ for some $c, d \in(0,2 \alpha)$.

Then, $\left\{x_{n}\right\},\left\{y_{n}\right\},\left\{z_{n}\right\}$, and $\left\{u_{n}\right\}$ converge strongly to $z_{0}=P_{\Omega} x_{0}$.

Proof. In Theorem 3.1, take $M=\partial \delta_{C}: H \rightarrow 2^{H}$, where $\delta_{C}: H \rightarrow[0, \infty]$ is the indicator function of $C$. It is well known that the subdifferential $\partial \delta_{C}$ is a maximal monotone operator. Then, problem (1.7) is equivalent to problem (4.1) and the resolvent operator $J_{M, \lambda_{n}}=P_{C}$ for all $n \in \mathbf{N}$. This completes the proof.

Next, we give a strong convergence theorem for finding a common element of the set of solutions of an equilibrium problem, the set of solutions of a variational inclusion and the set of common fixed points of a finite family of quasi-nonexpansive and Lipschitz mappings. In order to do this, let us assume that

(B3) for each $x \in H$ and $r>0$, there exists a bounded subset $D_{x} \subseteq C$ and $y_{x} \in C$ such that for any $z \in C \backslash D_{x}$,

$$
F\left(z, y_{x}\right)+\frac{1}{r}\left\langle y_{x}-z, z-x\right\rangle<0
$$

Theorem 4.2. Let $C$ be a nonempty closed convex subset of a real Hilbert space $H$, let $F: C \times C \rightarrow R$ be a bifunction satisfying (A1)-(A5), let $A: H \rightarrow H$ be an $\alpha$-inverse strongly monotone mapping, let $M: H \rightarrow 2^{H}$ be a maximal monotone mapping, and let $\left\{T_{i}\right\}_{i=1}^{N}$ be a finite family of quasinonexpansive and $L_{i}$-Lipschitz mappings of $C$ into itself. Assume that $\Omega:=\bigcap_{i=1}^{N} F\left(T_{i}\right) \cap \operatorname{EP}(F) \cap$ $I(A, M) \neq \emptyset$ and either (B1) or (B3) holds. Let $W_{n}$ be the $W$-mapping generated by $T_{1}, T_{2}, \ldots, T_{N}$ and 
$\beta_{n, 1}, \beta_{n, 2}, \ldots, \beta_{n, N}$. For an initial point $x_{0} \in H$ with $C_{1}=C$ and $x_{1}=P_{C_{1}} x_{0}$, let $\left\{x_{n}\right\},\left\{y_{n}\right\},\left\{z_{n}\right\}$, and $\left\{u_{n}\right\}$ be sequences generated by

$$
\begin{aligned}
& F\left(u_{n}, y\right)+\frac{1}{r_{n}}\left\langle y-u_{n}, u_{n}-x_{n}\right\rangle \geq 0, \quad \forall y \in C, \\
& y_{n}=\alpha_{n} x_{n}+\left(1-\alpha_{n}\right) W_{n} u_{n}, \\
& z_{n}=J_{M, \lambda_{n}}\left(y_{n}-\lambda_{n} A y_{n}\right), \\
& C_{n+1}=\left\{z \in C_{n}:\left\|z_{n}-z\right\| \leq\left\|y_{n}-z\right\| \leq\left\|x_{n}-z\right\|\right\}, \\
& x_{n+1}=P_{C_{n+1}} x_{0}, \quad \forall n \in \mathbf{N},
\end{aligned}
$$

where $\left\{\alpha_{n}\right\} \subset[0, a]$ for some $a \in[0,1),\left\{r_{n}\right\} \subset[b, \infty)$ for some $b \in(0, \infty)$, and $\left\{\lambda_{n}\right\} \subset[c, d]$ for some $c, d \in(0,2 \alpha)$.

Then, $\left\{x_{n}\right\},\left\{y_{n}\right\},\left\{z_{n}\right\}$, and $\left\{u_{n}\right\}$ converge strongly to $z_{0}=P_{\Omega} x_{0}$.

Proof. In Theorem 3.1, take $\varphi(x)=\delta_{C}(x)$, for all $x \in H$. Then problem (1.3) reduces to the equilibrium problem (1.5).

Remark 4.3. Theorem 3.1 improves and extends the main results in $[4,13]$ and the corresponding results.

\section{Acknowledgments}

The authors would like to thank the referee for the valuable suggestions on the manuscript. The authors were supported by the Commission on Higher Education, the Thailand Research Fund, and the Graduate School of Chiang Mai University.

\section{References}

[1] W. R. Mann, "Mean value methods in iteration," Proceedings of the American Mathematical Society, vol. 4, pp. 506-510, 1953.

[2] A. Genel and J. Lindenstrauss, "An example concerning fixed points," Israel Journal of Mathematics, vol. 22, no. 1, pp. 81-86, 1975.

[3] S. Reich, "Weak convergence theorems for nonexpansive mappings in Banach spaces," Journal of Mathematical Analysis and Applications, vol. 67, no. 2, pp. 274-276, 1979.

[4] K. Nakajo and W. Takahashi, "Strong convergence theorems for nonexpansive mappings and nonexpansive semigroups," Journal of Mathematical Analysis and Applications, vol. 279, no. 2, pp. 372379, 2003.

[5] G. Bigi, M. Castellani, and G. Kassay, "A dual view of equilibrium problems," Journal of Mathematical Analysis and Applications, vol. 342, no. 1, pp. 17-26, 2008.

[6] F. Flores-Bazán, "Existence theorems for generalized noncoercive equilibrium problems: the quasiconvex case," SIAM Journal on Optimization, vol. 11, no. 3, pp. 675-690, 2000.

[7] J.-W. Peng, Y.-C. Liou, and J.-C. Yao, "An iterative algorithm combining viscosity method with parallel method for a generalized equilibrium problem and strict pseudocontractions," Fixed Point Theory and Applications, vol. 2009, Article ID 794178, 21 pages, 2009.

[8] L.-C. Ceng and J.-C. Yao, "A hybrid iterative scheme for mixed equilibrium problems and fixed point problems," Journal of Computational and Applied Mathematics, vol. 214, no. 1, pp. 186-201, 2008.

[9] E. Blum and W. Oettli, "From optimization and variational inequalities to equilibrium problems," The Mathematics Student, vol. 63, no. 1-4, pp. 123-145, 1994. 
[10] P. L. Combettes and S. A. Hirstoaga, "Equilibrium programming in Hilbert spaces," Journal of Nonlinear and Convex Analysis, vol. 6, no. 1, pp. 117-136, 2005.

[11] S. D. Flåm and A. S. Antipin, "Equilibrium programming using proximal-like algorithms," Mathematical Programming, vol. 78, no. 1, pp. 29-41, 1997.

[12] A. N. Iusem and W. Sosa, "Iterative algorithms for equilibrium problems," Optimization, vol. 52, no. 3, pp. 301-316, 2003.

[13] A. Tada and W. Takahashi, "Weak and strong convergence theorems for a nonexpansive mapping and an equilibrium problem," Journal of Optimization Theory and Applications, vol. 133, no. 3, pp. 359-370, 2007.

[14] H. Bŕezis, "Operateur maximaux monotones," in Mathematics Studies, vol. 5, North-Holland, Amsterdam, The Netherlands, 1973.

[15] B. Lemaire, "Which fixed point does the iteration method select?" in Recent Advances in Optimization, vol. 452, pp. 154-167, Springer, Berlin, Germany, 1997.

[16] R. P. Agarwal, Y. J. Cho, and N.-J. Huang, “Sensitivity analysis for strongly nonlinear quasi-variational inclusions," Applied Mathematics Letters, vol. 13, no. 6, pp. 19-24, 2000.

[17] L.-C. Ceng, Q. H. Ansari, and J.-C. Yao, “On relaxed viscosity iterative methods for variational inequalities in Banach spaces," Journal of Computational and Applied Mathematics, vol. 230, no. 2, pp. 813-822, 2009.

[18] L.-C. Ceng, Q. H. Ansari, and J.-C. Yao, "Mann-type steepest-descent and modified hybrid steepestdescent methods for variational inequalities in Banach spaces," Numerical Functional Analysis and Optimization, vol. 29, no. 9-10, pp. 987-1033, 2008.

[19] L. C. Ceng, G. Y. Chen, X. X. Huang, and J.-C. Yao, "Existence theorems for generalized vector variational inequalities with pseudomonotonicity and their applications," Taiwanese Journal of Mathematics, vol. 12, no. 1, pp. 151-172, 2008.

[20] L.-C. Ceng, C. Lee, and J.-C. Yao, "Strong weak convergence theorems of implicit hybrid steepestdescent methods for variational inequalities," Taiwanese Journal of Mathematics, vol. 12, no. 1, pp. 227244, 2008.

[21] L.-C. Ceng, A. Petruşel, and J.-C. Yao, "Weak convergence theorem by a modified extragradient method for nonexpansive mappings and monotone mappings," Fixed Point Theory, vol. 9, no. 1, pp. 73-87, 2008.

[22] L.-C. Ceng, H.-K. Xu, and J.-C. Yao, "A hybrid steepest-descent method for variational inequalities in Hilbert spaces," Applicable Analysis, vol. 87, no. 5, pp. 575-589, 2008.

[23] L. C. Zeng, S. Schaible, and J.-C. Yao, "Hybrid steepest descent methods for zeros of nonlinear operators with applications to variational inequalities," Journal of Optimization Theory and Applications, vol. 141, no. 1, pp. 75-91, 2009.

[24] L.-C. Ceng and J.-C. Yao, "Relaxed viscosity approximation methods for fixed point problems and variational inequality problems," Nonlinear Analysis: Theory, Methods E Applications, vol. 69, no. 10, pp. 3299-3309, 2008.

[25] S. S. Chang, "Set-valued variational inclusions in Banach spaces," Journal of Mathematical Analysis and Applications, vol. 248, no. 2, pp. 438-454, 2000.

[26] P. Cholamjiak, "A hybrid iterative scheme for equilibrium problems, variational inequality problems, and fixed point problems in Banach spaces," Fixed Point Theory and Applications, vol. 2009, Article ID 719360, 18 pages, 2009.

[27] X. P. Ding, "Perturbed Ishikawa type iterative algorithm for generalized quasivariational inclusions," Applied Mathematics and Computation, vol. 141, no. 2-3, pp. 359-373, 2003.

[28] Y.-P. Fang and N.-J. Huang, " $H$-monotone operator and resolvent operator technique for variational inclusions," Applied Mathematics and Computation, vol. 145, no. 2-3, pp. 795-803, 2003.

[29] A. Kangtunyakarn and S. Suantai, "A new mapping for finding common solutions of equilibrium problems and fixed point problems of finite family of nonexpansive mappings," Nonlinear Analysis: Theory, Methods \& Applications, vol. 71, no. 10, pp. 4448-4460, 2009.

[30] A. Kangtunyakarn and S. Suantai, "Hybrid iterative scheme for generalized equilibrium problems and fixed point problems of finite family of nonexpansive mappings," Nonlinear Analysis: Hybrid Systems, vol. 3, no. 3, pp. 296-309, 2009.

[31] P. Kumam, "A hybrid approximation method for equilibrium and fixed point problems for a monotone mapping and a nonexpansive mapping," Nonlinear Analysis: Hybrid Systems, vol. 2, no. 4, pp. 1245-1255, 2008. 
[32] W. Nilsrakoo and S. Saejung, "Strong convergence theorems for a countable family of quasiLipschitzian mappings and its applications," Journal of Mathematical Analysis and Applications, vol. 356, no. 1, pp. 154-167, 2009.

[33] J.-W. Peng, Y. Wang, D. S. Shyu, and J.-C. Yao, “Common solutions of an iterative scheme for variational inclusions, equilibrium problems, and fixed point problems," Journal of Inequalities and Applications, vol. 2008, Article ID 720371, 15 pages, 2008.

[34] J.-W. Peng and J.-C. Yao, "A new hybrid-extragradient method for generalized mixed equilibrium problems, fixed point problems and variational inequality problems," Taiwanese Journal of Mathematics, vol. 12, no. 6, pp. 1401-1432, 2008.

[35] J.-W. Peng and J.-C. Yao, "A modified CQ method for equilibrium problems, fixed points and variational inequality," Fixed Point Theory, vol. 9, no. 2, pp. 515-531, 2008.

[36] A. Petrussel and J.-C. Yao, "An extragradient iterative scheme by viscosity approximation methods for fixed point problems and variational inequality problems," Central European Journal of Mathematics, vol. 7, no. 2, pp. 335-347, 2009.

[37] S. Plubtieng and W. Sriprad, "A viscosity approximation method for finding common solutions of variational inclusions, equilibrium problems, and fixed point problems in Hilbert spaces," Fixed Point Theory and Applications, vol. 2009, Article ID 567147, 20 pages, 2009.

[38] S. Schaible, J.-C. Yao, and L.-C. Zeng, "A proximal method for pseudomonotone type variational-like inequalities," Taiwanese Journal of Mathematics, vol. 10, no. 2, pp. 497-513, 2006.

[39] R. U. Verma, "A-monotonicity and applications to nonlinear variational inclusion problems," Journal of Applied Mathematics and Stochastic Analysis, vol. 2004, no. 2, pp. 193-195, 2004.

[40] L.-C. Zeng, S. M. Guu, and J.-C. Yao, "Hybrid approximate proximal point algorithms for variational inequalities in Banach spaces," Journal of Inequalities and Applications, vol. 2009, Article ID 275208, 17 pages, 2009.

[41] L. C. Zeng, L. J. Lin, and J.-C. Yao, "Auxiliary problem method for mixed variational-like inequalities," Taiwanese Journal of Mathematics, vol. 10, no. 2, pp. 515-529, 2006.

[42] L. C. Zeng and J.-C. Yao, "A hybrid extragradient method for general variational inequalities," Mathematical Methods of Operations Research, vol. 69, no. 1, pp. 141-158, 2009.

[43] S.-S. Zhang, J. H. Lee, and C. K. Chan, "Algorithms of common solutions to quasi variational inclusion and fixed point problems," Applied Mathematics and Mechanics, vol. 29, no. 5, pp. 571-581, 2008.

[44] G. Marino and H.-K. Xu, "Weak and strong convergence theorems for strict pseudo-contractions in Hilbert spaces," Journal of Mathematical Analysis and Applications, vol. 329, no. 1, pp. 336-346, 2007.

[45] C. Martinez-Yanes and H.-K. Xu, "Strong convergence of the CQ method for fixed point iteration processes," Nonlinear Analysis: Theory, Methods \& Applications, vol. 64, no. 11, pp. 2400-2411, 2006.

[46] W. Takahashi, Nonlinear Functional Analysis, Yokohama, Yokohama, Japan, 2000.

[47] S. Atsushiba and W. Takahashi, "Strong convergence theorems for a finite family of nonexpansive mappings and applications," Indian Journal of Mathematics, vol. 41, no. 3, pp. 435-453, 1999.

[48] K. Shimoji and W. Takahashi, "Strong convergence to common fixed points of infinite nonexpansive mappings and applications," Taiwanese Journal of Mathematics, vol. 5, no. 2, pp. 387-404, 2001.

[49] W. Takahashi, "Weak and strong convergence theorems for families of nonexpansive mappings and their applications," Annales Universitatis Mariae Curie-Skłodowska. Sectio A, vol. 51, no. 2, pp. 277-292, 1997.

[50] W. Takahashi, Convex Analysis and Approximation of Fixed Points, vol. 2, Yokohama, Yokohama, Japan, 2000. 